\title{
NOTE ON MAXIMAL ALGEBRAS
}

\section{G. HOCHSCHILD}

Introduction. It has been shown in a previous paper [3 $]^{1}$ that every algebra $A$ with radical $R$, such that $A / R$ is separable, is a homomorphic image of a certain maximal algebra which is determined to within an isomorphism by $A / R$, the $A / R$-module (two-sided) $R / R^{2}$, and the index of nilpotency of $R$. Furthermore, some indication was given of how the structure of maximal algebras can be determined in simple cases.

Here, we wish to give a further illustration by describing a rather wide class of maximal and primary algebras whose structure will be shown to resemble that of crossed products, in certain respects. In fact, we shall impose a certain normality condition and then trace the consequences of a few simple facts of the noncommutative Galois theory.

An algebra $B$ over the field $F$, with radical $R$, is called primary if it has an identity element and if $B / R$ is simple. As is well known, ${ }^{2} B$ is then isomorphic with a Kronecker product $F_{m} \times C$, where $F_{m}$ denotes the full matrix algebra of degree $m$ over $F$, and where $C$ is completely primary, in the sense that it has an identity element and that the quotient of $C$ by its radical is a division algebra over $F$. We are concerned with primary algebras $B$ for which this division algebra (which is determined to within an isomorphism by $B$ ) is normal over $F$, in the sense of the noncommutative Galois theory. ${ }^{3}$ This will be the case if and only if the center $Z$ of $B / R$ is a separable normal extension field of $F$ and every automorphism of $Z$ over $F$ is induced by an automorphism of $B / R$.

A completely primary algebra $C$ with radical $S$ will henceforth be called quasinormal if $C / S$ is normal over $F$. If $\phi$ is an isomorphism of $F_{m} \times C$ onto $B$ then $\phi$ maps the radical $F_{m} \times S$ onto the radical $R$ of $B$, and $B / R$ is isomorphic with $F_{m} \times C / S$. Therefore, if $C$ is quasinormal over $F$, then $B / R$ is automatically separable over $F$. By [3], $B$ has then a maximal related extension $B^{*}$ which is evidently primary. Moreover, it is easily seen that $B^{*} \approx F_{m} \times C^{*}$, where $C^{*}$ is the maximal related extension of $C$, and is quasinormal over $F$. Finally, the naturál extension to $B^{*}$ of a homomorphism of $C^{*}$ onto $C$ is a homomorphism of $B^{*}$ onto $B$. From these facts it is evident

Received by the editors October 11, 1948.

${ }^{1}$ Numbers in brackets refer to the bibliography at the end of the paper.

2 See, for instance, [2, chap. 2, \$9].

See [1] and [5]. 
that, without loss of generality, we may confine our attention to quasinormal algebras.

1. Representation in the radical. Let $B$ be quasinormal over $F$, and let $R$ be its radical. By Wedderburn's theorem and the quasinormality we have $B=A+R$, where $A$ is a normal division algebra over $F$. Moreover, it is easy to see that the identity element of $A$ coincides with the identity element of $B$. Hence, if $R$ is regarded in the natural fashion as a two-sided $A$-module, the identity element of $A$ acts as the identity operator on either side.

As in $[3, \S 4]$, we make a direct decomposition of the $A$-module $R$ in the form $R=T+R^{2}$. The submodule $T$ (which is isomorphic with the $A$-module $R / R^{2}$ ) is decomposed further into a direct sum of (twosided) simple submodules. Now it is easily seen that every simple $A$-module over $F$ is an operator homomorphic image of the Kronecker product $A \times A$ with respect to $F$, regarded in the natural fashion as a two-sided $A$-module. By applying either the noncommutative Galois theory, or the classical representation theory for simple algebras in conjunction with the theory of field composites (for $Z \times Z$, where $Z$ is the center of $A$ ), we conclude that the simple components of the $A$-module $A \times A$ are of dimension 1 over $A .^{4}$ It follows that every simple $A$-module is of dimension 1 over $A$, and if $u$ is a generator we have $u \cdot a=\sigma\{a\} \cdot u$, for every $a \in A$, where $\sigma$ is an automorphism of $A$ over $F$. Two such simple modules are operator isomorphic if and only if the corresponding automorphisms differ only by an inner automorphism of $\boldsymbol{A}$.

It follows that there is a set of automorphisms $\sigma_{1}, \cdots, \sigma_{\varepsilon}$ (not necessarily distinct) of $A$ over $F$ which are determined uniquely up to inner automorphisms, ${ }^{b}$ and a corresponding decomposition, $T$ $=A \cdot u_{1}+\cdots+A \cdot u_{\mathrm{a}}$ of $T$ into simple submodules, such that, for each index $i$ and every $a \in A, u_{i} \cdot a=\sigma_{i}\{a\} \cdot u_{i}$.

2. The standard maximal algebras. Let $B$ be as in $\$ 1$. We construct a maximal related extension of $B$ from $A$ and $T$ by the process described in $[3,84]$. If $B$ is already maximal the resulting "standard algebra" will be isomorphic with $B$.

The radical of the algebra to be constructed is the linearly direct sum of the "powers" $T^{(k)}, k=1, \cdots, n$, where $n$ is the index of nil$\$ 4$ ].

4 A proof of this, in a terminology close to the present one, will be found in [4,

- This because the simple components of a semisimple module are unique to within an automorphism of the whole module, as follows, for instance, from the evident fact that they are isomorphic images of the factors in a composition series. 
potency of $R$. We have $T^{(1)}=T$, and generally $T^{(k+1)}$ is the Kronecker product $T^{(k)} \times T$, which means that in the elementary products $u \times v$, where $u \in T^{(k)}$ and $v \in T$, we identify $u \cdot a \times v$ with $u \times a \cdot v$ for every $a \in A$, and define the module operations such that $a \cdot(u \times v)=a \cdot u \times v$ and $(u \times v) \cdot a=u \times v \cdot a$. There is a natural multiplication $\left(T^{(k)}, T^{(l)}\right)$ $\rightarrow T^{(k+l)}$, for $k+l \leqq n$, and $\left(T^{(k)}, T^{(l)}\right) \rightarrow(0)$ for $k+l>n$, by means of which the multiplication in the standard algebra is defined.

It is easy to see that the partial products $A \cdot u_{i} \times A \cdot u_{j}$ are simple $A$-modules and may be represented in the form $A \cdot u$, with $u \cdot a$ $=\sigma_{i} \sigma_{j}\{a\} \cdot u$, for every $a \in A$. From this and $\$ 4$ of [3], it can be seen immediately that the standard algebra may be described as follows:

Its elements are polynomials of total degree not greater than $n$, with coefficients in $A$, in the freely noncommuting "variables" $u_{1}, \cdots, u_{s}$. The elements are multiplied like ordinary polynomials, subject to the commutation rules $u_{i} a=\sigma_{i}\{a\} u_{i}$, and all the terms of degree greater than $n$ are omitted. We denote this algebra by $A\left(u_{1}, \cdots, u_{s}\right)_{n}$. Our result is the following:

THEOREM. Every quasinormal maximal algebra $B$ with radical $R$ is isomorphic with a standard "polynomial algebra" $B / R\left(u_{1}, \cdots, u_{s}\right)_{n}$, where $s$ is the number of simple components of the $B / R$-module $R / R^{2}$, and $n$ is the index of nilpotency of $R$. To each $u_{i}$ there belongs an automorphism $\sigma_{i}$ of $B / R$ over $F$ such that $u_{i} a=\sigma_{i}\{a\} u_{i}$ for every $a \in B / R$. These automorphisms are determined by $B$ to within inner automorphisms of $B / R$. Two standard algebras with the same coefficient rings $B / R$ are isomorphic if and only if their autmorphisms $\sigma_{i}$ and $\tau_{i}$ can be so indexed that, for each $i, \sigma_{i} \tau_{i}^{-1}$ is an inner automorphism of $B / R$.

As in $[3, \S 6]$, we obtain the following corollary:

CoRollary. If $B$ is quasinormal and if $R / R^{2}$ is a simple $B / R$ module, then $B$ is maximal and is isomorphic with a standard algebra $B / R\left(u_{\sigma}\right)_{n}$. Two such algebras are isomorphic if and only if the corresponding automorphisms differ only by an inner automorphism of $B / R$.

In general, every primary algebra satisfying the normality condition laid down in the introduction is a homomorphic image of an algebra of the type $F_{m} \times A\left(u_{1}, \cdots, u_{s}\right)_{n}$, the kernel of the homomorphism consisting of "polynomials" in which the "constant" terms and the terms of degree 1 in the $u_{i}$ 's are absent.

\section{BIBLIOGRAPHY}

1. N. Cartan, Theorie de Galois pur les corps non commutatifs, Ann. Ecole Norm. vol. 64 (1947) pp. 59-77. 
2. M. Deuring, Algebren, Ergebnisse der Mathematik und ihrer Grenzgebiete, vol. 4, Berlin, Springer, 1935.

3. G. Hochschild, On the structure of algebras with nonzero radical, Bull. Amer. Math. Soc. vol. 53 (1947) pp. 369-377.

4. - Double vector spaces over division rings, Amer. J. Math. vol. 51 (1949) pp. 443-460.

5. N. Jacobson, A note on division rings, Amer. J. Math. vol. 49 (1947) pp. 27-36.

UNIVERSITY OF ILLINOIS

\section{ON THE RADICAL OF A LIE ALGEBRA}

\section{HARISH-CHANDRA}

Let $\mathfrak{R}$ be a Lie algebra over a field $K$ of characteristic zero. For any $X \in \mathbb{R}$ we denote, as usual, the linear mapping $Y \rightarrow[X, Y]$ of $\mathbb{R}$ into itself by ad $X$. Let $\Gamma$ be the radical of $\mathfrak{R}$. Consider the set $\mathfrak{R}$ consisting of all $N \in \Gamma$ such that ad $N$ is nilpotent. It was shown in a recent paper ${ }^{1}$ that $\mathfrak{N}$ is the unique maximal nilpotent ideal ${ }^{2}$ of $\mathfrak{R}$. Further if $D$ is a derivation of $\Gamma$ then $D \Gamma \subset \Re$.

For any $X, Y, Z \in \mathbb{R}$ put $B(X, Y)=s p(\operatorname{ad} X$ ad $Y)$ and $T(X, Y, Z)$ $=s p(\operatorname{ad}[X, Y] \operatorname{ad} Z)$. Then $B(X, Y)$ is a symmetric bilinear form on $\&$ while $T(X, Y, Z)$ is a skewsymmetric trilinear form. It is easily verified that they are both invariant under all derivations of $\mathfrak{R}$, that is,

$$
\begin{gathered}
B(D X, Y)+B(X, D Y)=0, \\
T(D X, Y, Z)+T(X, D Y, Z)+T(X, Y, D Z)=0
\end{gathered}
$$

for any derivation $D$ and $X, Y, Z \in \mathbb{R}$.

An ideal $\mathfrak{M}$ in $\mathfrak{R}$ is called characteristic if $D \mathfrak{M} \subset \mathfrak{M}$ for every derivation $D$ of $\&$. Our first theorem may now be stated as follows:

Theorem 1. An element $X$ of $\&$ belongs to the radical $\Gamma$ if and only if $T(X, Y, Z)=0$ for all $Y, Z \in \mathbb{R}^{3}$

As an immediate corollary we get the following:

Received by the editors October 4, 1948 and, in revised form, November 11, 1948.

1 Ann. of Math. vol. 50 (1949) p. 68.

2 My attention has been drawn to a paper by Malcev (Bull. Acad. Sci. URSS. vol. 9 (1945) pp. 329-356) where it is shown that $\mathfrak{R}$ is an ideal.

- Since $T(X, Y, Z)=-T(Z, Y, X)$ this condition is clearly equivalent to $B(X, Y)$ $=0$ for all $Y \in \mathbb{R}^{\prime}=[R, \&]$. Professor Jacobson has kindly brought it to my notice that this theorem is contained in Cartan's thesis p. 109. 